\title{
Pediatric Readmissions and the Quality of Hospital-to-Home Transitions
}

\author{
Morgan Congdon, MD, MPH${ }^{1,2 *}$, Andrew S Kern-Goldberger, MD', Jessica K Hart, MD ${ }^{1,2}$
}

'Division of General Pediatrics, The Children's Hospital of Philadelphia, Philadelphia, Pennsylvania; ${ }^{2}$ Department of Pediatrics, The Perelman School of Medicine at The University of Pennsylvania, Philadelphia, Pennsylvania.

ince 2012, when the Centers for Medicare \& Medicaid Services (CMS) began linking financial penalties to hospitals with excessive readmissions for adult patients, researchers have questioned the extent to which pediatric readmissions can be used as a reliable quality measure. Compared with readmissions among adult patients, readmissions among pediatric patients are relatively uncommon. Furthermore, few (approximately 2\%) qualify as potentially preventable, and pediatric readmission rates remain largely unchanged despite targeted attempts to prevent reutilization. ${ }^{1,2}$ Nonetheless, state Medicaid agencies have continued to reduce reimbursement for hospitals based on available readmissions metrics, most commonly the Potentially Preventable Readmissions (PPR) algorithm. ${ }^{1}$

In this issue of the Journal of Hospital Medicine, Auger et $\mathrm{al}^{3}$ performed a retrospective study to explore four existing metrics of pediatric hospital readmissions for their ability to identify preventable and unplanned readmissions. Investigators examined 30-day readmissions $(n=1,125)$ from 2014-2016 across multiple subspecialties, and classified readmissions by their preventability and unplanned status with use of a validated chart abstraction tool. Using the results of chart abstraction as the gold standard, investigators calculated the sensitivity and specificity, as well as estimated the positive and negative predictive values, of each readmissions metric. Auger and colleagues found that none of the four readmissions metrics could reliably assess preventability, and that only one metric reliably predicted unplanned hospital readmissions. Specifically, the commonly used PPR algorithm was estimated to have a positive predictive value of $13.0 \%-35.5 \%$ across a prevalence range of $10 \%-30 \%$. This means that in a hospital where $10 \%$ of readmissions are truly preventable, the PPR will be wrong approximately $87 \%$ of the time. Tying payments to this metric is difficult to justify.

The authors highlighted the policy implications of the PPR falling short in its ability to identify preventable and unplanned pediatric readmissions. A good quality measure should be consistently reliable, and neither the PPR nor other measures

*Corresponding Author: Morgan Congdon MD, MPH; Email: congdonm@ email.chop.edu; Telephone: 215-906-1261; Twitter: @CongdonMorgan.

Received: August 13, 2020; Revised: August 18, 2020;

Accepted: August 19, 2020

๑ 2020 Society of Hospital Medicine DOI 10.12788/jhm.3525 studied meets this benchmark. Yet the findings lead to a broader conclusion: if most pediatric readmissions are not preventable, if there is no reliable way of measuring preventability, and if we have not demonstrated the ability to change patient trajectories away from reutilization, then perhaps the sun has set on using readmissions as a comprehensive quality measure for hospital-based care.

So how, then, should the hospital-to-home transition be evaluated? The paradigm of pediatric value of care is shifting to incorporate family-centered perspectives into consideration of quality measures. ${ }^{2}$ There has to be a balance between healthcare costs and outcomes that affect families; measures should take into account issues such as patient and caregiver anxiety and time away from work. ${ }^{2}$ Moreover, because social determinants of health and medical complexity strongly influence readmission rates ${ }^{4,5}$ focus should be placed on redirecting resources toward patients and families with significant medical, social, and financial needs as they transition home from the hospital. While measures of healthcare equity are currently lacking, the overall quality and equity of pediatric care transitions could be enhanced by looking beyond the narrow lens of readmission rates to incorporate actual needs assessments of families.

In summary, Auger and colleagues identified deficits in existing readmission metrics-but creating a solution that is meaningful to all stakeholders will be more complex than simply identifying a better metric. Family-centered quality metrics show promise in creating value in pediatric care within an equitable health system, but long-term evaluation of these metrics is necessary.

Disclosure: The authors have nothing to disclose.

\section{References}

1. Auger KA, Harris JM, Gay JC, et al. Progress (?) toward reducing pediatric readmissions. J Hosp Med. 2019;14(10):618-621. https://doi.org/10.12788/ jhm. 3210

2. Forrest $\mathrm{CB}$, Silber JH. Concept and measurement of pediatric value. Acad Pediatr. 2014;14(5 Suppl):S33-S38. https://doi.org/10.1016/j.acap.2014.03.013

3. Auger K, Ponti-Zins M, Statile A, Wesselkamper K, Haberman B, Hanke S. Performance of pediatric readmission measures. J Hosp Med. 2020;15:723726. https://doi.org/10.12788/jhm.3521

4. Berry JG, Hall DE, Kuo DZ, et al. Hospital utilization and characteristics of patients experiencing recurrent readmissions within children's hospitals. JAMA. 2011;305(7):682-690. https://doi.org/10.1001/jama.2011.122

5. Beck $A F$, Huang $B$, Simmons JM, et al. Role of financial and social hardships in asthma racial disparities. Pediatrics. 2014;133(3):431-439. https://doi. org/10.1542/peds.2013-2437 